\title{
Radioresistance of chordoma cells is associated with the ATM/ATR pathway, in which RAD51 serves as an important downstream effector
}

\author{
CHAO ZHANG, BING WANG, LEI LI, YAWEI LI, PENGZHI LI and GUOHUA LV \\ Department of Spine Surgery, The Second Xiangya Hospital of \\ Central South University, Changsha, Hunan 410011, P.R. China
}

Received July 21, 2016; Accepted May 5, 2017

DOI: $10.3892 /$ etm.2017.4736

\begin{abstract}
Surgery followed by radiotherapy is the standard treatment for chordomas, which are a rare but low-grade type of bone cancer arising from remnants of the embryonic notochord. However, disease recurrence following radiotherapy is common, most likely due to endogenous DNA repair mechanisms that promote cell survival upon radiation strikes. The ataxia telangiectasia mutated/ataxia telangiectasia mutated and Rad3 related (ATM/ATR)-mediated pathway has a critical role in DNA repair mechanisms; however, it has rarely been investigated in chordomas. In the present study, the expression of signal molecules related to the ATM/ATR pathway in chordoma tissues and adjacent normal tissues were initially examined using immunohistochemistry and western blot analysis. Chordoma U-CH1 and U-CH2 cells were subsequently used to investigate cell responses to ionizing radiation and the potential protective actions mediated by the ATM/ATR pathway. Phosphorylated (p)-ATM, p-ATR, $\gamma$-H2A histone family, member X (H2AX) and RAD51 were significantly upregulated in chordoma tissues relative to adjacent normal tissues $(\mathrm{P}<0.05)$. No significant reductions were observed in the viability of $\mathrm{U}-\mathrm{CH} 1$ and $\mathrm{U}-\mathrm{CH} 2$ cells following exposure to low-dose (1 and 2 Gy) radiation. Radiation (1 and 2 Gy) triggered a significant upregulation in p-ATM, $\gamma$-H2AX and RAD51 expression in $\mathrm{U}-\mathrm{CH} 1$ cells $(\mathrm{P}<0.05)$, as well as a significant upregulation in p-ATM, p-ATR and RAD51 levels in $\mathrm{U}-\mathrm{CH} 2$ cells $(\mathrm{P}<0.05)$. RAD51 knockdown increased the responses of both $\mathrm{U}-\mathrm{CH} 1$ and $\mathrm{U}-\mathrm{CH} 2$ cells to 1 Gy radiation, as evidenced by the significantly decreased cell viability and increased apoptosis rate $(\mathrm{P}<0.05)$. Collectively, the results of
\end{abstract}

Correspondence to: Professor Guohua Lv, Department of Spine Surgery, The Second Xiangya Hospital of Central South University, 139 Middle Renmin Road, Changsha, Hunan 410011, P.R. China E-mail: lguohua2017@163.com

Key words: radioresistance, chordomas, ataxia telangiectasia mutated/ataxia telangiectasia mutated and Rad3 related, RAD51 the present study indicated that radioresistance of chordoma cells is associated with the ATM/ATR pathway, in which RAD51 serves as an important downstream effector. Thus, RAD51 presents a promising therapeutic target for improving the outcome of radiotherapy treatment in chordomas.

\section{Introduction}

Chordomas are rare primary malignant bone tumors, accounting for $\sim 5 \%$ of malignant bone tumors and $\sim 17 \%$ of primary malignant bone tumors of the axial skeleton $(1,2)$. Chordomas are typically low-grade, poorly differentiated and locally invasive, which may be associated with the fact that they originate from embryonic notochord remnants in the axial skeleton (2). Although several chemotherapeutic agents have been reported to induce positive responses in individual cases, chordomas are usually resistant to chemotherapy, and limited options exist for systemic therapy (2). The standard treatment for chordomas is local therapy in the form of surgery and radiotherapy (3). However, the recurrence rate is high following surgery and radiotherapy; chordomas typically recur within 29-43 months and the 5-year progression-free survival rate is $23-65 \%$, with a median overall survival of 6 years (2).

Several lines of evidence have demonstrated that DNA repair mechanisms are employed by cancer cells to resist ionizing radiation, resulting in cancer recurrence and final radiotherapy failure (4-6). It has been reported that the protein kinases, ataxia telangiectasia mutated (ATM) and ATM and Rad3 related (ATR), are major regulators in the DNA damage response to radiation $(4,5)$. An increased number of ATM and ATR foci have been observed in damaged DNA following $\gamma$-ray and carbon ion irradiation (6). Individuals with the disease ataxia telangiectasia, in which no ATM protein is expressed, are more sensitive to radiation (7). Furthermore, the administration of pharmacologic ATM/ATR kinase inhibitors has been reported to radiosensitize lung cancer cells (6). A previous study demonstrated that irradiation-induced DNA damage increases the kinase activities of ATM and ATR (8). Activated ATM and ATR phosphorylate a broad range of substances to induce cell cycle checkpoints, initiate DNA repair and control cell survival or apoptosis (8). In the DNA repair action, ATM/ATR triggers phosphorylation of the histone variant, $\mathrm{H} 2 \mathrm{~A}$ histone 
family, member $\mathrm{X}(\mathrm{H} 2 \mathrm{AX})$, forming $\gamma-\mathrm{H} 2 \mathrm{AX}$ (8). It also has a key role in the recruitment and accumulation of DNA repair proteins at sites of DNA damage (8). RAD51 is an evolutionarily conserved recombinase downstream of the ATM/ATR pathway $(9,10)$. With the assistance of breast cancer susceptibility gene 2 (BRCA2) protein and other factors, RAD51 is recruited to the DNA damage sites and polymerized onto the resection-generated single-strand DNA ends, which leads to the formation of a nucleoprotein filament that promotes strand invasion and exchange between homologous DNA sequences $(9,10)$. Strand invasion is followed by the initiation of DNA synthesis from the $3^{\prime}$ end of the invading strand and eventual DNA repair $(9,10)$. Although ATM/ATR-mediated DNA repair has been examined in multiple cancer types, little data was available regarding its role in chordomas $(9,10)$. The aim of the present study was to explore the potential effect of the ATM/ATR pathway in chordoma cells that were subjected to ionizing radiation, and to elucidate the mechanisms underlying poor radiotherapy outcomes and radiation treatment failure.

\section{Materials and methods}

Tissue samples and ethics statement. A total of 26 pairs of chordoma and adjacent normal tissue samples were obtained from patients (age, 28-65 years old; 14 female patients and 12 male patients; mean age, $48.30 \pm 12.90$ years old) who underwent tumor resection at the Third Xiangya Hospital of Central South University (Changsha, China) between January 2012 and December 2015. A number of the patients (19/26) showed symptoms such as headache, hearing impairments, impotence and amenorrhea prior to the surgery. None of the recruited patients underwent preoperative radiotherapy or chemotherapy and were histopathologically confirmed to have primary chordoma cancer. Patients that had underwent preoperative radiotherapy or chemotherapy were excluded from the present study. The present study was approved by the Ethics Committee of Xiangya School of Medicine (Changsha, China), and all patients provided written informed consent.

Immunohistochemistry (IHC). Tissue samples were fixed at room temperature in 10\% buffered formalin for 12-24 h and embedded in paraffin. Paraffin-embedded tumor and normal tissue samples were processed into $4-\mu \mathrm{m}$ sections, deparaffinized with xylene and rehydrated in a series of ethanol washes. Antigen retrieval was performed in a pressure cooker at $121^{\circ} \mathrm{C}(100 \mathrm{kpa}$ ) for $15 \mathrm{~min}$ with $0.01 \mathrm{M}$ citrate buffer (pH 6.0; Sigma-Aldrich; Merck KGaA, Darmstadt, Germany). To block any potential non-specific binding of the secondary antibody, sections were incubated in $10 \%$ normal goat serum (Merck KGaA) for 30 min at room temperature. Sections were subsequently incubated with primary antibodies including anti-phosphorylated (p)-ATM (1:5,000; ab81292; Abcam, Cambridge, MA, USA), anti-p-ATR (1:1,000; ab178407; Abcam), anti- $\gamma$-H2AX (1:500; ab26350; Abcam), anti-BRCA2 (1:500; ab27976; Abcam) and anti-RAD51 (1:500; ab111568; Abcam), at $4^{\circ} \mathrm{C}$ overnight, before incubation with secondary biotin-conjugated antibodies (B-2763 and 65-6140; Thermo Fisher Scientific, Inc., Waltham, MA, USA) with a 1:1,000 dilution at $37^{\circ} \mathrm{C}$ for $1 \mathrm{~h}$. Finally, sections were lightly counterstained using hematoxylin at $37^{\circ} \mathrm{C}$ for $1 \mathrm{~min}$. The sections were observed using LeicaTCS-SP5 microscopy (magnification, $\mathrm{x} 400$; Leica instrument Co., Ltd, Beijing, China) and a high-resolution CoolSNAP CCD camera (Photometrics Inc., Tucson, AZ, USA).

Western blotting. Tissue and cell lysates were prepared using lysis buffer (50 mM Tris-HCl, $\mathrm{pH} 7.4,150 \mathrm{mM} \mathrm{NaCl}$, $10 \mathrm{mM} \mathrm{NaF}, 2 \mathrm{mM}$ EDTA, $1 \% \mathrm{NP}-40$ and $0.1 \%$ sodium dodecyl sulfate). Protein concentration was measured using a bicinchoninic acid protein assay kit (Beyotime Institute of Biotechnology, Shanghai, China). Equal amounts of protein $(20 \mu \mathrm{g})$ were separated by $10 \%$ SDS-PAGE and electroblotted onto a nitrocellulose membrane. The membrane was blocked with $5 \%$ non-fat milk overnight at $4^{\circ} \mathrm{C}$ and subsequently incubated with primary antibodies against p-ATM $(1: 1,000$; ab81292; Abcam), anti-p-ATR (1:700; ab178407; Abcam), anti- $\gamma$-H2AX (1:400; ab26350; Abcam), anti-BRCA2 (1:500; ab27976; Abcam), anti-RAD51 (1:500; ab111568; Abcam) and $\beta$-actin (1:800; sc-47778; Santa Cruz Biotechnology, Inc., Santa Cruz, CA, USA) at $37^{\circ} \mathrm{C}$ for $2 \mathrm{~h}$. The membrane was then incubated with horseradish peroxidase-conjugated secondary antibodies (1:2,000; A0168 and A9169; Sigma-Aldrich; Merck $\mathrm{KGaA}$ ) at $37^{\circ} \mathrm{C}$ for $1 \mathrm{~h}$. Immunoreactive bands in the membrane were developed using enhanced chemiluminescent substrates (Pierce; Thermo Fisher Scientific, Inc.). $\beta$-actin protein expression was used as a loading control. Relative quantitation was determined using the AlphaView 3.0 system (ProteinSimple, San Jose, CA, USA).

Cell culture and treatments. U-CH1 and U-CH2 chordoma cell lines were obtained from the American Type Culture Collection (Manassas, VA, USA) and cultured at $37^{\circ} \mathrm{C}$ in Iscove's modified Dulbecco's medium (IMDM)/RPMI (4:1; Gibco; Thermo Fisher Scientific, Inc., Waltham, MA, USA) supplemented with $10 \%$ heat inactivated fetal bovine serum (FBS; Invitrogen; Thermo Fisher Scientific, Inc.), $100 \mathrm{U} / \mathrm{ml}$ penicillin and $100 \mu \mathrm{g} / \mathrm{ml}$ streptomycin. Cells were cultured to exponential growth, for approximately $\sim 36 \mathrm{~h}$. Subsequently the exponentially grown cells were cultured in the medium without FBS at $37^{\circ} \mathrm{C}$ overnight and subjected to different doses of ionizing radiation $(0,1,2,4$ or $8 \mathrm{~Gy})$ using an Image Guided Radiotherapy (IGRT) system (Elekta Compact, Stockholm, Sweden). In detail, the exponentially growing cells were cultured in the serum-free medium and irradiated with $6 \mathrm{MeV}$ photons $/ 20 \mathrm{~cm}$ focal surface distance at a dose rate of $2 \mathrm{~Gy} / \mathrm{min}$ at room temperature. Following irradiation, the cells were cultivated at $37^{\circ} \mathrm{C}$ for $72 \mathrm{~h}$, which allowed sufficient time for the generation of irradiation-induced cytotoxicity. A subset of $\mathrm{U}-\mathrm{CH} 1$ and $\mathrm{U}-\mathrm{CH} 2$ cells were subjected to RAD51 knockdown prior to exposure to ionizing radiation. For these cells, RAD51 expression was silenced by transfection with small hairpin RNA (shRNA) targeting RAD51 (GenePharma Co., Ltd., Shanghai, China) using the Lipofectamine 2000 (Invitrogen; Thermo Fisher Scientific, Inc., Carlsbad, California, USA), according to the manufacturer's protocol. shRNA-RAD51 was transformed to small interfering (si)RNA-RAD51 (sense, 5'-GGGUACCUUUAGGCCAGAATT-3'; antisense 5'-UUC UGGCCUAAAGGUACCCTT-3') in cells. shRNA-RAD51 
was dissolved in the Lipofectamine ${ }^{\circledR} 2000$ with the final concentration of $60 \mathrm{nM}$ piror to transfection into $\mathrm{U}-\mathrm{CH} 1$ and $\mathrm{U}-\mathrm{CH} 2$ cells.

Cell viability assay. Following irradiation, cell viability was assessed using a Cell Counting Kit-8 detection kit (CCK-8; Sigma-Aldrich; Merck KGaA). The cells were seeded at a density of $1 \times 10^{5}$ cells/well in 96-well plates and cultured with $90 \mu \mathrm{IMDM} / \mathrm{RPMI}$ culture medium and $10 \mu \mathrm{l}$ CCK-8 reagent at $37^{\circ} \mathrm{C}$ for $1 \mathrm{~h}$. The optical density was measured at $490 \mathrm{~nm}$ for each well using an enzyme immunoassay analyzer.

Colony formation assay. Following irradiation, U-CH1 and $\mathrm{U}-\mathrm{CH} 2$ cells were seeded on soft agar $\left(3 \times 10^{7}\right.$ cells/well in 6 -well plates) and incubated at $37^{\circ} \mathrm{C}$ for 1 week to allow colony growth. Colonies were stained with crystal violet at $37^{\circ} \mathrm{C}$ for $20 \mathrm{~min}$ and images of the stained plates were captured. Each colony $>75 \mathrm{~mm}$ in diameter or containing $>50$ cells was counted as one positive colony. The colony formation rate was calculated using the following equation: Number of positive colonies/number of cells originally seeded.

Reverse transcription-quantitative polymerase chain reaction $(R T-q P C R)$. Total RNA was extracted from cells using TRIzol reagent (Thermo Fisher Scientific, Inc.). The RNA was treated with DNA-free DNase and reverse transcribed for cDNA synthesis using a PrimeScript RT Reagent Kit (Takara Bio, Inc., Otsu, Japan) according to the manufacturer's instruction's. qPCR analysis was performed using a SYBR-Green Reaction kit (Takara Biotechnology Co., Ltd., Dalian, China) on an MJ Mini Gradient Thermal Cycler Real-Time PCR machine (Bio-Rad Laboratories, Inc., Hercules, CA, USA). The following primers were used: RAD51, forward 5'-CAACCCATTTCACGGTTAGAGC-3' and reverse 5'-TTCTTTGGCGCATAGGCAACA-3'; and GAPDH, forward 5'-TGGGCTACACTGAGCACCAG-3' and reverse 5'-GGGTGTCGCTGTTGAAGTCA-3'. The thermocycling conditions were as follows: $94^{\circ} \mathrm{C}$ for $5 \mathrm{~min}$ followed by 35 cycles of $30 \mathrm{sec}$ at $94^{\circ} \mathrm{C}, 30 \mathrm{sec}$ at $55^{\circ} \mathrm{C}$ and $50 \mathrm{sec}$ at $70^{\circ} \mathrm{C}$, and a final extension step of $72^{\circ} \mathrm{C}$ for $10 \mathrm{~min}$. Expression data were uniformly normalized to GAPDH as an internal control, and the relative expression levels were evaluated using the $2^{-\Delta \Delta \mathrm{Cq}}$ method (11).

Flow cytometric analysis of apoptotic cells. Apoptosis was detected using Annexin V-fluorescein isothiocyanate (FITC)/propidium iodide (PI) staining followed by flow cytometry. Following irradiation, the cells were washed twice with cold PBS and incubated with annexin V-FITC and PI (Annexin V-FITC/PI Apoptosis Detection Kit; Beyotime Institute of Biotechnology) at room temperature for $15 \mathrm{~min}$ in the dark. The cells were collected and analyzed using a FACSCanto II flow cytometer (BD Biosciences, Franklin Lakes, NJ, USA) and ModFit LT software version 2.0 (Verity Software House, Inc., Topsham, ME, USA) was used.

Statistical analysis. All statistical analyses were performed using SPSS 17.0 software from SPSS Inc., (Chicago, IL, USA). Data were presented as the mean \pm standard deviation. Data was analyzed using one-way analysis of variance followed by a Scheffe Post-hoc test. $\mathrm{P}<0.05$ was considered to indicate a statistically significant difference.

\section{Results}

Expression of signaling molecules relative to the ATM/ATR pathway in chordoma tissues and adjacent normal tissues. The expression levels of signaling molecules were mapped relative to the ATM/ATR pathway in chordoma tissues and adjacent normal tissues. ATM and ATR are apical DNA damage signaling kinases that phosphorylate substantial downstream molecules $(4,5)$. IHC detection revealed that p-ATM staining was present in the nucleus and cytoplasm of adjacent normal cells of chordomas in all patients; however, staining was markedly more abundant in the cancer cells (Fig. 1). p-ATR was expressed in a few of the adjacent normal cells of chordomas, whereas it was expressed in the nucleus and cytoplasm of the majority of chordoma cells (Fig. 1). H2AX is a variant of nucleosome core histone $\mathrm{H} 2 \mathrm{~A}$, and phosphorylated $\mathrm{H} 2 \mathrm{AX}$ is defined as $\gamma$-H2AX (8). $\gamma$-H2AX was observed in a small number of normal cells in some patients and in relatively more chordoma cell nuclei in 20 of the 26 patients (Fig. 1). BRCA2 and RAD51 are downstream molecules in the ATM/ATR pathway, responsible for DNA repair $(9,10)$. IHC images revealed no obvious difference in BRCA2 expression between chordoma cells and adjacent normal cells (Fig. 1). Very little RAD51 staining was observed in adjacent normal cells of chordomas, whereas in the cancer tissues RAD51 staining was widely and substantially distributed in the nucleus and cytoplasm.

Western blotting was subsequently performed to quantify the expression of these proteins and to identify the difference in their expression levels between chordoma and adjacent normal tissues. The results demonstrated a significant increase in the protein expression levels of p-ATM, p-ATR, $\gamma$-H2AX and RAD51 in chordoma tissues compared with adjacent normal tissues $(\mathrm{P}<0.05$; Fig. 2). However, no significant difference was observed in the expression of BRCA2 between chordoma tissues and adjacent normal tissues (data not shown).

Alterations in cell viability and colony formation rate of chordomas following exposure to ionizing radiation. To investigate the effect of irradiation on chordomas, the viability and colony formation rate of $\mathrm{U}-\mathrm{CH} 1$ and $\mathrm{U}-\mathrm{CH} 2$ cells were assessed following exposure to different doses of ionizing radiation. Irradiation with 4 or 6 Gy significantly decreased U-CH1 cell viability compared with 0 Gy $(\mathrm{P}<0.05$; Fig. $3 \mathrm{~A})$, and $\mathrm{U}-\mathrm{CH} 2$ cell viability was significantly reduced following exposure to 6 Gy radiation $(\mathrm{P}<0.05$; Fig. $3 \mathrm{~A})$. However, low dose radiation, 1 or $2 \mathrm{~Gy}$, had no significant inhibitory effect on $\mathrm{U}-\mathrm{CH} 1$ and $\mathrm{U}-\mathrm{CH} 2$ cell viability. Irradiation with 2, 4 or 6 Gy significantly reduced the colony formation rate of $\mathrm{U}-\mathrm{CH} 1$ cells compared with 0 Gy $(\mathrm{P}<0.05$; Fig. 3B); however, 1 Gy had only moderate effects in this respect. Irradiation with 4 or 6 Gy also significantly decreased the colony formation rate of $\mathrm{U}-\mathrm{CH} 1$ cells compared with 0 Gy $(\mathrm{P}<0.05$; Fig. 3B). In contrast, the effect of irradiation at 1 and 2 Gy was insignificant.

ATM/ATR signal in chordoma cells in response to low doses of radiation. The expression levels of signaling molecules in 


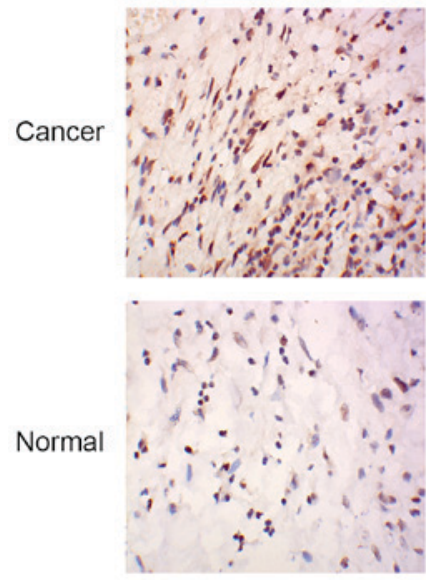

p-ATM
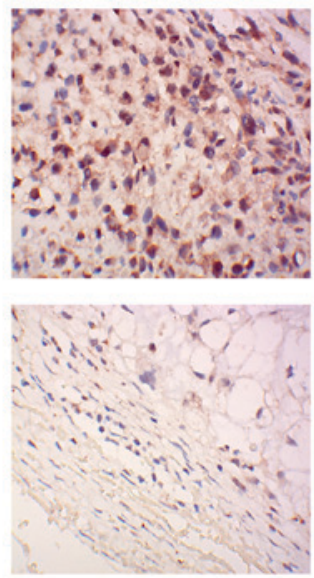

p-ATR
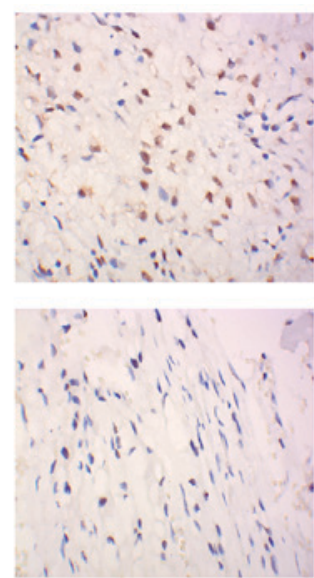

$\gamma-\mathrm{H} 2 \mathrm{AX}$

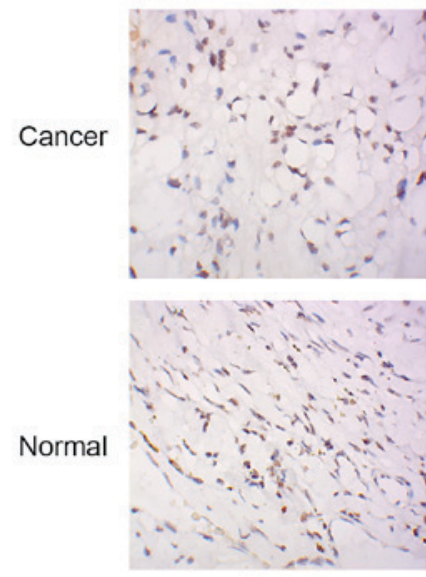

BRCA2

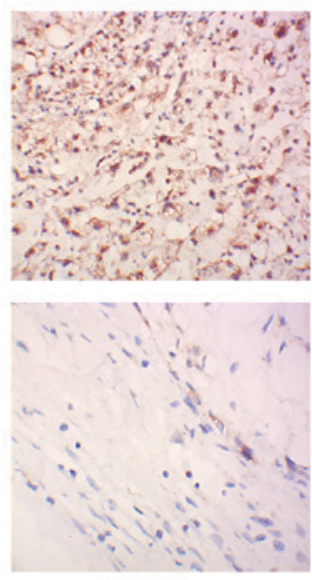

RAD51

Figure 1. Immunohistochemistry detection mapped the expression of signaling molecules relative to the ATM/ATR pathway in chordoma tissues and adjacent normal tissues stained with hematoxylin. p-ATM was moderately expressed in the nucleus and cytoplasm of adjacent normal cells, whereas it was highly expressed and largely accumulated in the nuclei of chordoma cells. p-ATR was expressed at a low level in adjacent normal cells; however, it was highly expressed in the nuclei and cytoplasm of chordoma cells. A small increase in $\gamma$-H2AX expression was observed in the nuclei of chordoma cells compared with adjacent normal tissues. No obvious difference in BRCA2 expression was observed between chordoma and adjacent normal cells. RAD51 expression was low in normal cells, whereas RAD51 staining was widely and substantially distributed in the nuclei and cytoplasm of chordomas. ATM, ataxia telangiectasia mutated; ATR, ATM and Rad3 related; p, phosphorylated; $\gamma$-H2AX, phosphorylated $\mathrm{H} 2 \mathrm{~A}$ histone family, member X; BRCA2, breast cancer susceptibility gene 2. Magnification, $\mathrm{x} 400$.

ATM/ATR cascades were detected in U-CH1 and U-CH2 cells following exposure to low doses of radiation. In U-CH1 cells, 1 or 2 Gy radiation significantly upregulated the expression levels of p-ATM, $\gamma$-H2AX and RAD51 proteins compared with control cells $(\mathrm{P}<0.05$; Fig. 4), but not those of p-ATR and BRCA2. In U-CH2 cells, p-ATM, p-ATR and RAD51 were significantly upregulated compared with control cells $(\mathrm{P}<0.05$; Fig. 4) following exposure to 1 or 2 Gy radiation. $\gamma$ - $\mathrm{H} 2 \mathrm{AX}$ expression was slightly increased, and BRCA2 maintained stable expression in $\mathrm{U}-\mathrm{CH} 2$ cells following irradiation with 1 or $2 \mathrm{~Gy}$.

Silencing RAD51 expression in $\mathrm{U}-\mathrm{CH} 1$ and $\mathrm{U}-\mathrm{CH} 2$ cells before radiation. To investigate whether RAD51 has an important role in inducing the radioresistance of chordoma cells, RAD51 was transiently silenced via transfection with shRNA-RAD51. RT-qPCR revealed that 1 Gy irradiation significantly increased RAD51 expression in both U-CH1 and $\mathrm{U}-\mathrm{CH} 2$ cells compared with untreated cells $(\mathrm{P}<0.05$;
Fig. 5A). RAD51 knockdown significantly decreased RAD51 expression in U-CH1 and U-CH2 cells compared with control groups $(\mathrm{P}<0.05$; Fig. $5 \mathrm{~A})$. U-CH1 and $\mathrm{U}-\mathrm{CH} 2$ cells with RAD51 knockdown maintained a low expression level of RAD51 following irradiation ( $\mathrm{P}<0.05$; Fig. $5 \mathrm{~A})$. Western blotting demonstrated that $1 \mathrm{~Gy}$ radiation significantly increased RAD51 expression in both U-CH1 and U-CH2 cells compared with control cells $(\mathrm{P}<0.05$; Fig. 5B).

Silencing RAD51 sensitizes $U-C H 1$ and $U-C H 2$ cells to ionizing radiation. Silencing RAD51 had no significant effect on U-CH1 and U-CH2 cell viability; however, cell viability was significantly decreased following the combination of silencing of RAD51 and exposure to 1 Gy radiation $(\mathrm{P}<0.05$; Fig. 6A). RAD51 knockdown or irradiation with $1 \mathrm{~Gy}$ alone had no significant effect on the apoptosis rate of U-CH1 or U-CH2 cells; however, RAD51 knockdown followed by 1 Gy irradiation significantly increased their apoptosis rate $(\mathrm{P}<0.01$; Fig. 6B). The colony formation rates of $\mathrm{U}-\mathrm{CH} 1$ and $\mathrm{U}-\mathrm{CH} 2$ 

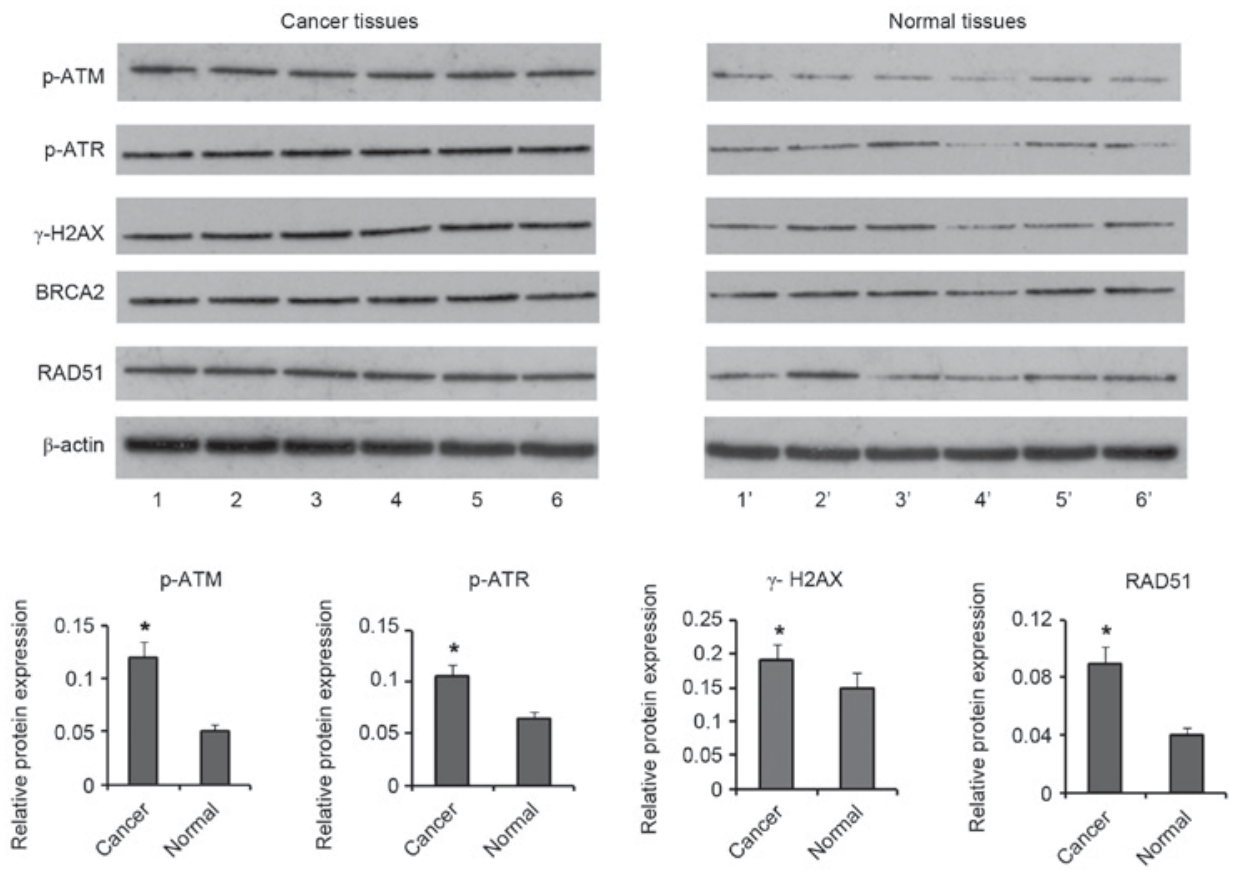

Figure 2. Western blotting was used to quantify signaling molecules relative to the ATM/ATR pathway in chordoma and adjacent normal tissues. The results revealed a significant increase in the protein expressions of p-ATM, p-ATR, $\gamma$-H2AX and RAD51 in chordoma tissues compared with adjacent normal tissues. "P $<0.05$ vs. normal tissues. ATM, ataxia telangiectasia mutated; ATR, ATM and Rad3 related; p, phosphorylated; $\gamma$-H2AX, phosphorylated H2A histone family, member X.
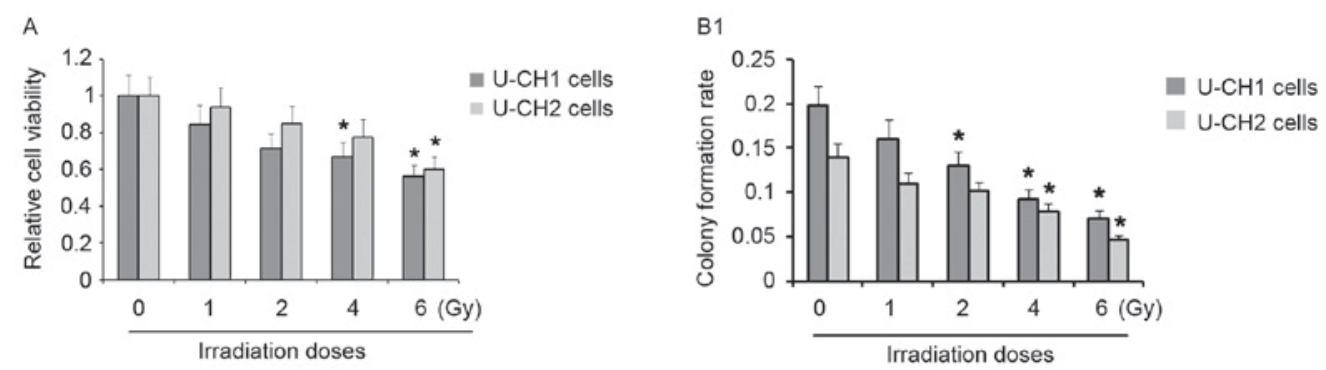

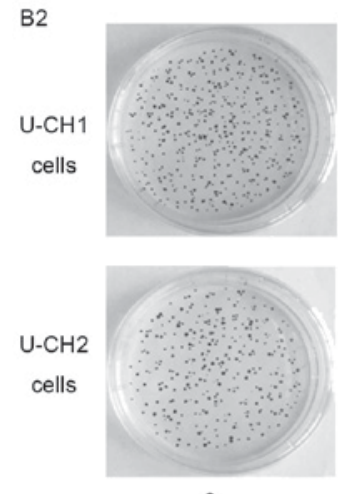

0
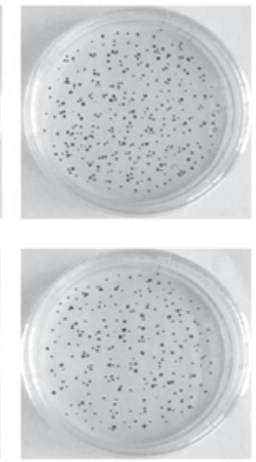

1
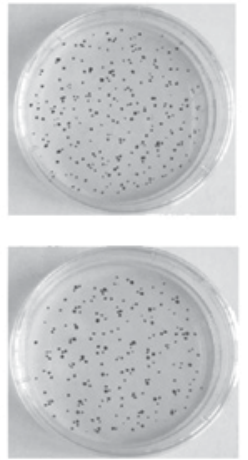
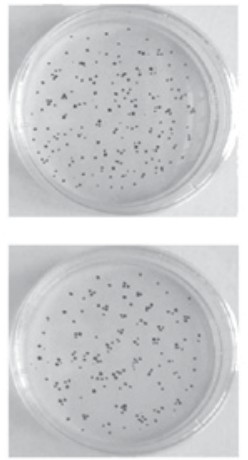
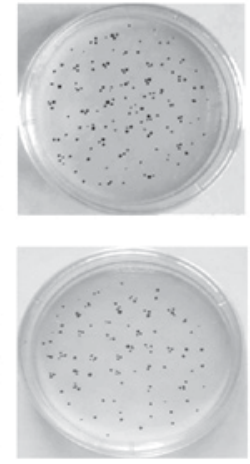

$6(\mathrm{~Gy})$

Figure 3. The effect of ionizing radiation on cell viability and colony formation rate of chordoma cells. (A) Cell viability and (B1 and B2) colony formation rate of $\mathrm{U}-\mathrm{CH} 1$ and $\mathrm{U}-\mathrm{CH} 2$ cells were evaluated following exposure to different doses of ionizing radiation. High-dose radiation significantly reduced cell viability and colony formation rate, whereas the effects of low-dose radiation were insignificant. " $\mathrm{P}<0.05$ vs. 0 Gy radiation.

cells were not significantly affected by treatment with 1 Gy radiation or RAD51 knockdown alone, whereas RAD51 knockdown followed by 1 Gy irradiation induced a significant decrease in colony formation rate compared with the control $(\mathrm{P}<0.05$; Fig. 6C).

\section{Discussion}

Radiotherapy is a standard treatment approach for chordomas; however, a few chordoma cells are not swept out following irradiation due, in part, to the acquirement of radioresistance (3). 

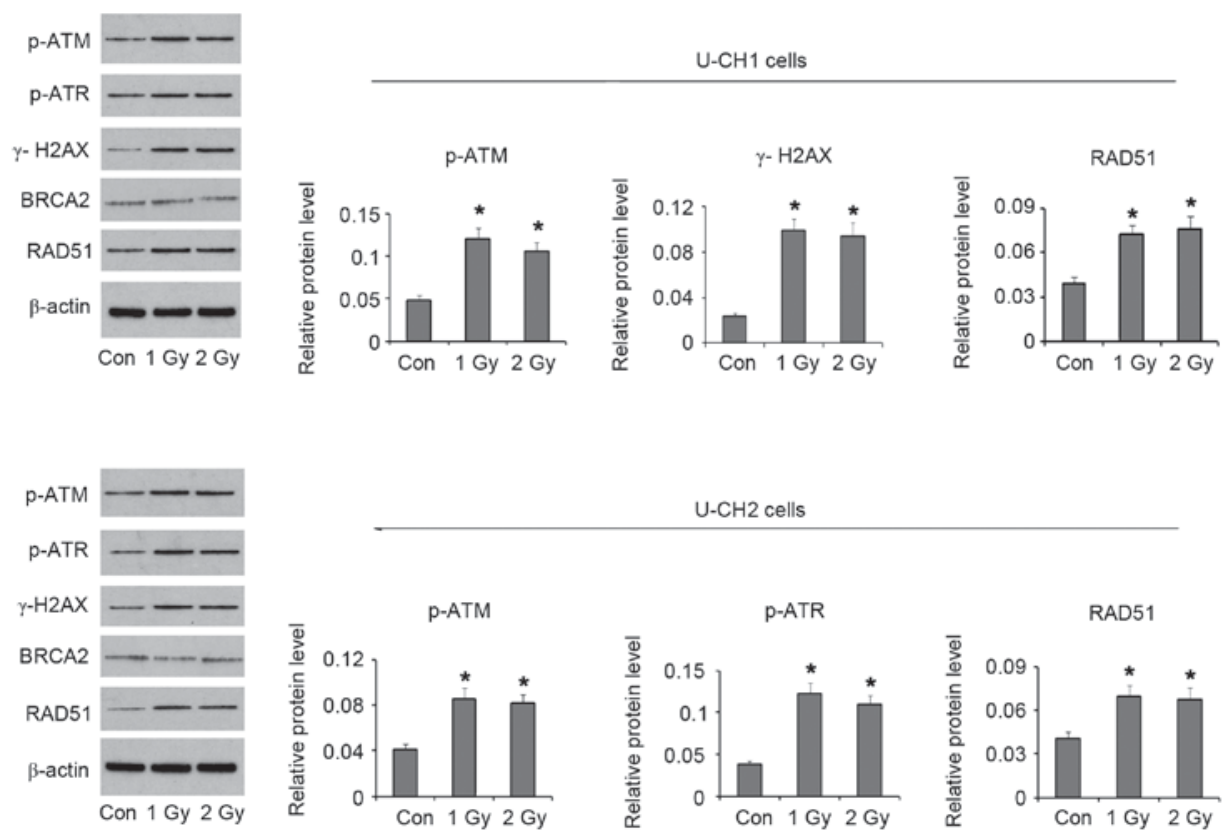

Figure 4. ATM/ATR signaling in chordoma cells exposed to low-dose radiation. In U-CH1 cells, 1 and 2 Gy radiation led to an upregulation in the expression of p-ATM, $\gamma$-H2AX and RAD51 proteins, but not p-ATR and BRCA2. p-ATM, p-ATR and RAD51 were upregulated in U-CH2 cells compared with Con cells, whereas $\gamma$-H2AX expression level increased only marginally and BRCA2 remained unchanged. "P $<0.05$ vs. Con. ATM, ataxia telangiectasia mutated; ATR, ATM and Rad3 related; p, phosphorylated; $\gamma$-H2AX, phosphorylated H2A histone family, member X; BRCA2, breast cancer susceptibility gene 2; Con, untreated control cells.

A
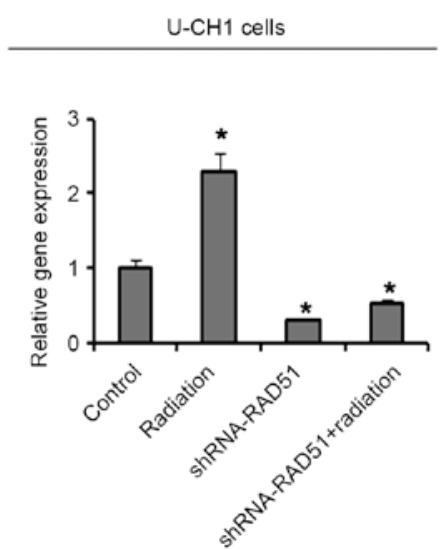

B

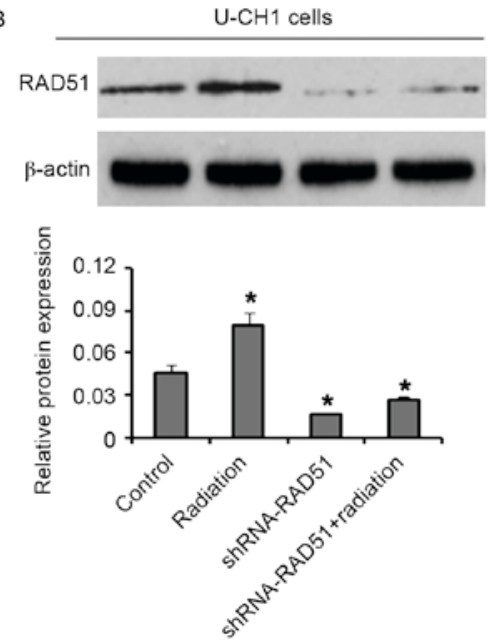

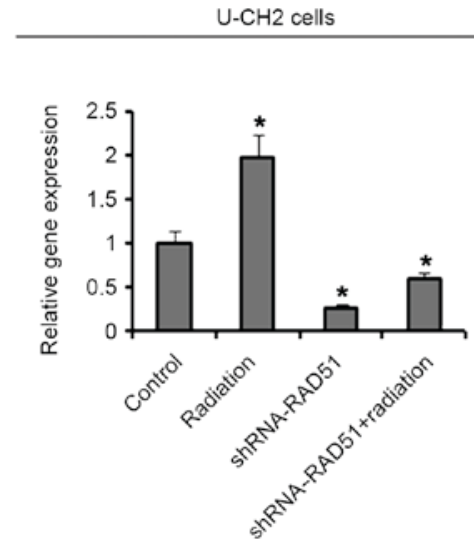
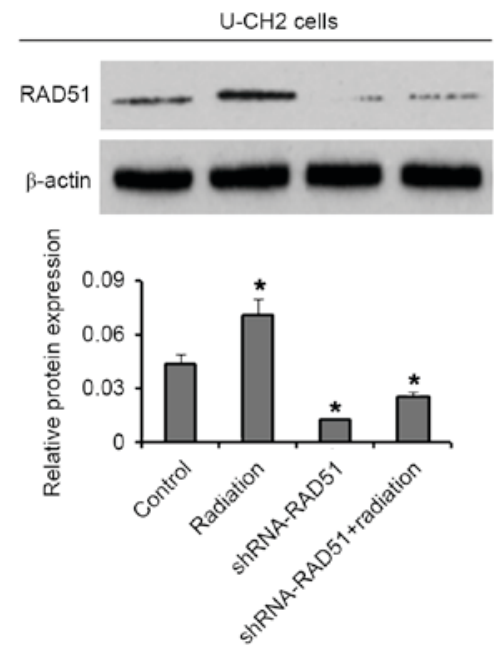

Figure 5. Silencing RAD51 expression in U-CH1 and U-CH2 cells prior to irradiation. RAD51 was transiently silenced by transfecting cells with shRNA-RAD51. (A) Reverse transcription-quantitative polymerase chain reaction and (B) western blotting were performed and it was demonstrated that RAD51 expression was significantly downregulated in U-CH1 and U-CH2 cells following transfection. Subsequent exposure to 1 Gy radiation did not restore the RAD51 expression. ${ }^{*} \mathrm{P}<0.05$ vs. control. shRNA, short hairpin RNA. 
A

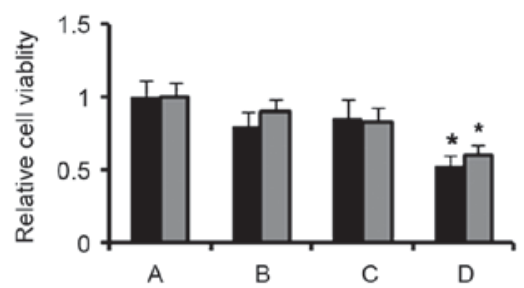

B
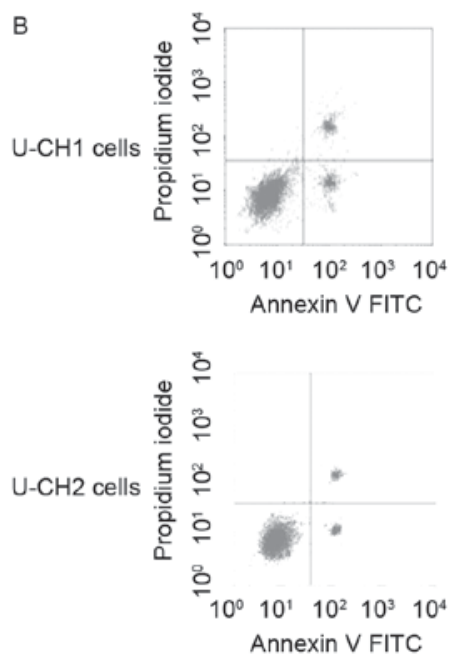

A
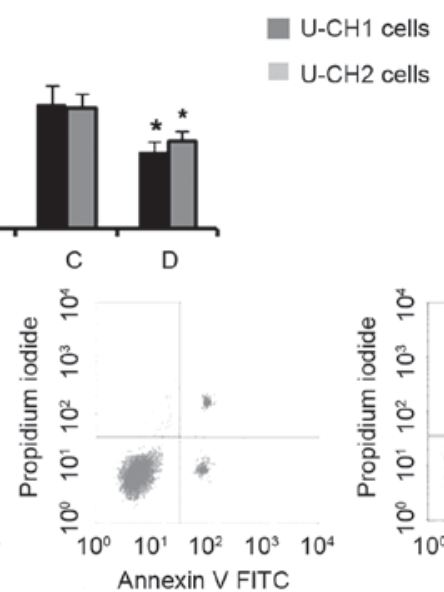

Treatment group:

A. Control

B. Radiation (1 Gy)

C. shRNA-RAD51

D. shRNA-RAD51 + radiation (1 Gy)
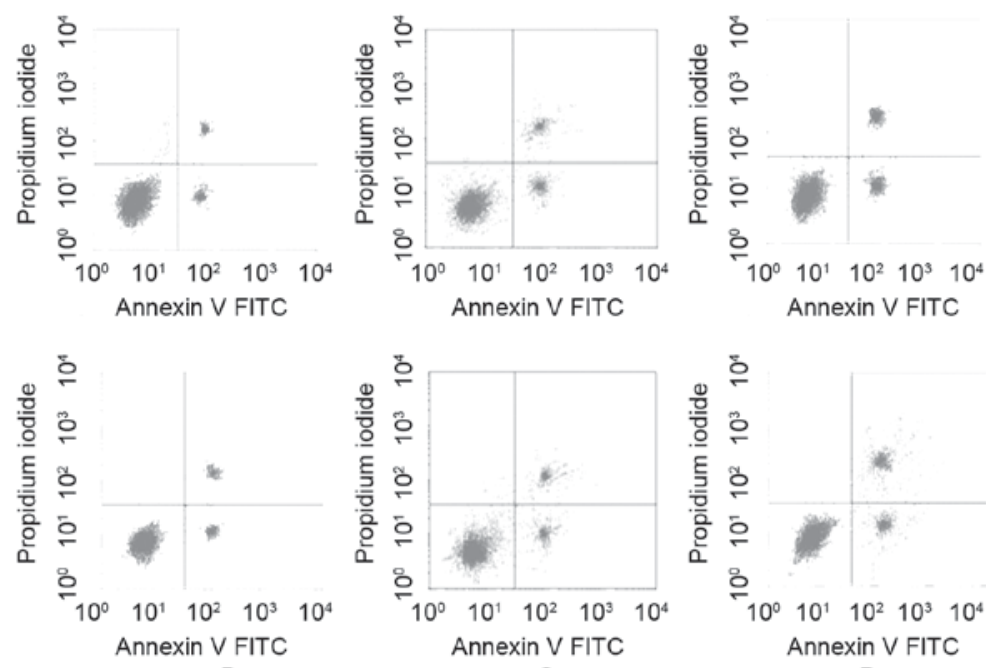

$B$

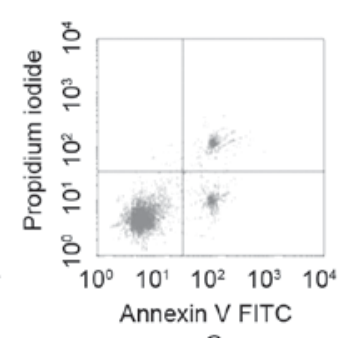

C

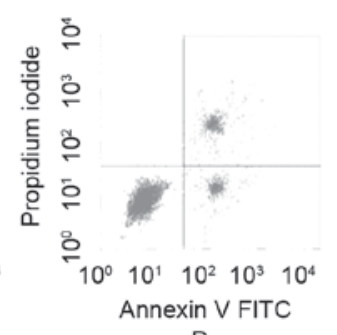

D

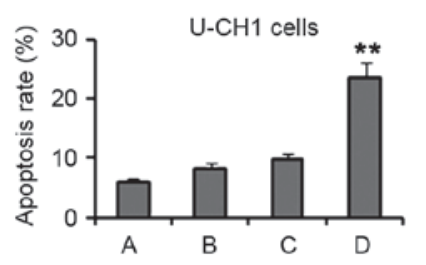

C

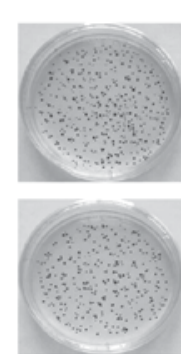

A

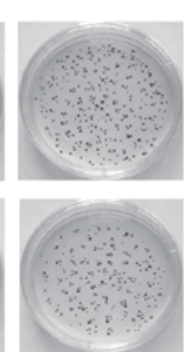

B

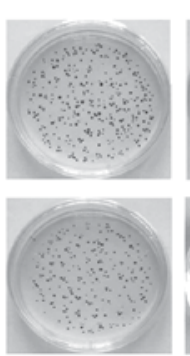

c

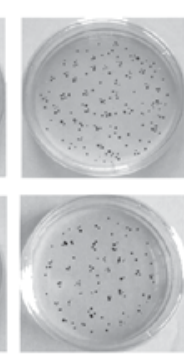

D
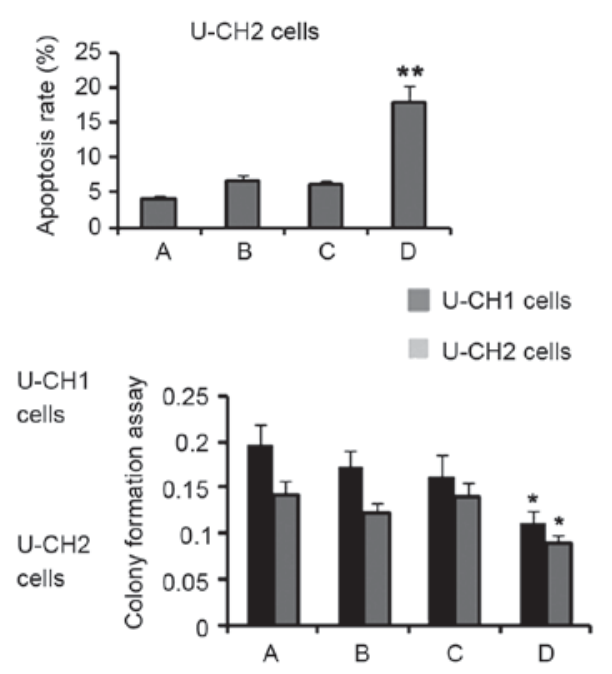

Figure 6. Silencing RAD51 sensitizes U-CH1 and U-CH2 cells to ionizing radiation. RAD51 was knocked down in U-CH1 and U-CH2 cells via shRNA transfection prior to $1 \mathrm{~Gy}$ irradiation. The (A) cell viability, (B) apoptosis rate and (C) colony formation rate of $\mathrm{U}-\mathrm{CH} 1$ and $\mathrm{U}-\mathrm{CH} 2$ cells were used to evaluate the sensitivity of the cells to low-dose irradiation. ${ }^{*} \mathrm{P}<0.05$ and ${ }^{* *} \mathrm{P}<0.01$ vs. control. shRNA, short hairpin RNA; FITC, fluorescein isothiocyanate.

It has been reported that irradiation induces DNA repair mechanisms, which serve as a protective action against apoptosis triggered by radiation-induced DNA damage (8). ATM/ATR are central modulators of DNA repair mechanisms in diverse cancer types upon ionizing radiation (4-6); however, little evidence is available to clarify the role of the ATM/ATR pathway in chordomas. The present study initially investigated the abundance and location of signal molecules related to the ATM/ATR pathway in chordomas and adjacent non-cancer tissues to elucidate the basic expression profile. The expression levels of p-ATM, p-ATR, $\gamma$-H2AX and RAD51 were revealed to be significantly higher in chordoma tissues than in adjacent non-cancer tissues. Notably, both chordomas and adjacent normal tissues were obtained from patients that had never been subjected to preoperative radiotherapy. As such, the observed upregulation of p-ATM, p-ATR, $\gamma$-H2AX and RAD51 in chordoma tissues was not a result of radiation. The underlying reason for this upregulation requires further investigation. This phenomenon may indicate that chordomas naturally possess potent DNA repair abilities mediated by the ATM/ATR pathway.

To understand the response to radiation, chordoma U-CH1 and $\mathrm{U}-\mathrm{CH} 2$ cells were subjected to different doses of ionizing radiation. Although there was a significant reduction in cell viability following exposure to high-dose radiation, the effect of low doses of radiation (e.g., 1 or $2 \mathrm{~Gy}$ ) on cell viability was insignificant. This suggests that chordoma cells are resistant to low-dose radiation. Considering that low-dose radiotherapy is 
extensively employed in clinical settings to minimize the side effects of radiation, it is highly possible that many chordoma cells survive following radiation treatment, resulting in the high recurrence of chordoma (12).

In the present study, it was demonstrated that some signaling molecules associated with the ATM/ATR pathway were upregulated after chordoma cells were exposed to 1 and 2 Gy radiation, which suggests that radiation may drive the onset of ATM/ATR-mediated DNA repair mechanisms. Notably, the changes in the expression levels of these signaling molecules were not entirely consistent between $\mathrm{U}-\mathrm{CH} 1$ and $\mathrm{U}-\mathrm{CH} 2$ cells. p-ATR was upregulated in $\mathrm{U}-\mathrm{CH} 2$ cells and not in $\mathrm{U}-\mathrm{CH} 1$ cells following irradiation; furthermore, $\gamma-\mathrm{H} 2 \mathrm{AX}$ was upregulated in $\mathrm{U}-\mathrm{CH} 1$ cells but not in $\mathrm{U}-\mathrm{CH} 2$ cells. ATR responds primarily to stalled replication forks where the generation of replication protein A-coated single-stranded DNA activates its kinase activity (13). Although ATM and ATR are associated in most cases, mechanisms of ATM kinase activation are different from that of ATR. The former predominantly depends on the signals arisen from the presence of double-strand breaks (13). In the present study, p-ATM was upregulated in $\mathrm{U}-\mathrm{CH} 1$ cells following irradiation, whereas p-ATR was not. $\gamma$-H2AX has been described as a highly specific and sensitive molecular marker for monitoring DNA double-strand breaks and its repair (13). The upregulation of $\gamma-\mathrm{H} 2 \mathrm{AX}$ in $\mathrm{U}-\mathrm{CH} 1$ cells therefore may support the activation of ATM in U-CH1 cells. Although no significant alteration in $\gamma$-H2AX expression was observed, U-CH2 cells exhibited elevated p-ATM and p-ATR following irradiation, suggesting that DNA repair actions mediated by both ATM and ATR were enhanced by radiation. This may explain why $\mathrm{U}-\mathrm{CH} 2$ exhibited a greater degree of radioresistance than U-CH1 following exposure to $4 \mathrm{~Gy}$ radiation.

Low-dose radiation upregulated p-ATM and RAD51 expression levels in both $\mathrm{U}-\mathrm{CH} 1$ and $\mathrm{U}-\mathrm{CH} 2$ cells, indicating that p-ATM and RAD51 have important roles in DNA repair mechanisms following radiation. Previous studies have demonstrated that ATM responds to radiation-induced DNA damage and then activates cell cycle checkpoints and promotes DNA repair $(6,14,15)$. If DNA lesions are able to be repaired in a timely and accurate manner, ATM-modulated signaling prevents cell cycle arrest and permits cell survival; if not, ATM initiates apoptotic pathways, such as that mediated by $\mathrm{p} 53$, to induce apoptosis $(16,17)$. Some controversy, therefore, exists as to whether it is beneficial to suppress ATM kinase activity in cancer therapy, and a few studies have reported that using specific inhibitors of ATM enhances the response of cancer cells to ionizing radiation and anti-cancer agents $(6,18)$. RAD51 is a key downstream molecule of the ATM/ATR pathway, responsible for DNA repair and promoting cell survival $(9,10)$. It has been reported that RAD51 knockdown via small interfering RNA transfection attenuates radioresistance or chemoresistance in cancer cells $(19,20)$. In the present study, RAD51 was knocked down in U-CH1 and U-CH2 cells, which effectively improved the cell response to low-dose radiation, suggesting that RAD51 serves as an important regulator contributing to the radioresistance of chordoma cells.

In conclusion, the results of the present study revealed that p-ATM, p-ATR, $\gamma$-H2AX and RAD51 are upregulated in chordoma tissues relative to adjacent normal tissues. Furthermore, 1 or 2 Gy radiation triggers an upregulation in p-ATM, $\gamma$-H2AX and RAD51 expression in U-CH1 cells and upregulates p-ATM, p-ATR and RAD51 expression levels in U-CH2 cells. Knocking down RAD51 increased the cellular responses of both $\mathrm{U}-\mathrm{CH} 1$ and $\mathrm{U}-\mathrm{CH} 2$ to 1 Gy radiation, as evidenced by the significant decrease in cell viability and increased apoptosis rate. These findings indicated that radioresistance in chordoma cells is associated with the ATM/ATR pathway, in which RAD51 serves as an important downstream effector. Based on these results, RAD51 may be a promising therapeutic target for improving the outcome of radiotherapy for the treatment of chordomas.

\section{References}

1. Tranesh G and Nassar A: Metastatic Chordoma: A diagnostic challenge on fine needle aspiration. Case Rep Pathol 2016: $2187290,2016$.

2. Gellner V, Tomazic PV, Lohberger B, Meditz K, Heitzer E, Mokry M, Koele W, Leithner A, Liegl-Atzwanger B and Rinner B: Establishment of clival chordoma cell line MUG-CC1 and lymphoblastoid cells as a model for potential new treatment strategies. Sci Rep 6: 24195, 2016.

3. Fujii R, Friedman ER, Richards J, Tsang KY, Heery CR, Schlom J and Hodge JW: Enhanced killing of chordoma cells by antibody-dependent cell-mediated cytotoxicity employing the novel anti-PD-L1 antibody avelumab. Oncotarget 7: 33498-33511, 2016.

4. Durisova K, Salovska B, Pejchal J and Tichy A: Chemical inhibition of DNA repair kinases as a promising tool in oncology. Biomed Pap Med Fac Univ Palacky Olomouc Czech Repub 160: 11-19, 2016.

5. Hein AL, Ouellette MM and Yan Y: Radiation-induced signaling pathways that promote cancer cell survival (review). Int J Oncol 45: 1813-1819, 2014.

6. Yang L, Liu Y, Sun C, Yang X, Yang Z, Ran J, Zhang Q, Zhang H, Wang $X$ and Wang $X$ : Inhibition of DNA-PKcs enhances radiosensitivity and increases the levels of ATM and ATR in NSCLC cells exposed to carbon ion irradiation. Oncol Lett 10: 2856-2864, 2015.

7. Vendetti FP, Lau A, Schamus S, Conrads TP, O'Connor MJ and Bakkenist CJ: The orally active and bioavailable ATR kinase inhibitor AZD6738 potentiates the anti-tumor effects of cisplatin to resolve ATM-deficient non-small cell lung cancer in vivo. Oncotarget 6: 44289-44305, 2015.

8. Ray A, Mir SN, Wani G, Zhao Q, Battu A, Zhu Q, Wang QE and Wani AA: Human SNF5/INI1, a component of the human SWI/SNF chromatin remodeling complex, promotes nucleotide excision repair by influencing ATM recruitment and downstream H2AX phosphorylation. Mol Cell Biol 29: 6206-6219, 2009.

9. Tsabar M, Mason JM, Chan YL, Bishop DK and Haber JE: Caffeine inhibits gene conversion by displacing Rad51 from ssDNA. Nucleic Acids Res 43: 6902-6918, 2015.

10. Velic D, Couturier AM, Ferreira MT, Rodrigue A, Poirier GG, Fleury F and Masson JY: DNA damage signalling and repair inhibitors: The long-sought-after achilles' heel of cancer. Biomolecules 5: 3204-3259, 2015.

11. Livak KJ and Schmittgen TD: Analysis of relative gene expression data using real-time quantitative PCR and the 2(-Delta Delta C(T)) method. Methods 25: 402-408, 2001.

12. Jin Q, Li XJ and Cao PG: MicroRNA-26b enhances the radiosensitivity of hepatocellular carcinoma cells by targeting EphA2. Tohoku J Exp Med 238: 143-151, 2016.

13. Amiard S, Charbonnel C, Allain E, Depeiges A, White CI and Gallego ME: Distinct roles of the ATR kinase and the Mre11-Rad50-Nbs1 complex in the maintenance of chromosomal stability in Arabidopsis. Plant Cell 22: 3020-3033, 2010.

14. Huang H, Fletcher L, Beeharry N, Daniel R, Kao G, Yen TJ and Muschel RJ: Abnormal cytokinesis after X-irradiation in tumor cells that override the G2 DNA damage checkpoint. Cancer Res 68: 3724-3732, 2008.

15. Zhong R, Xin R, Chen Z, Liang N, Liu Y, Ma S and Liu X: The role of deoxycytidine kinase $(\mathrm{dCK})$ in radiation-induced cell death. Int J Mol Sci 17: pii: E1939, 2016. 
16. Vici P,Di Benedetto A,Ercolani C,Pizzuti L, Di Lauro L, Sergi D, Sperati F, Terrenato I, Dattilo R, Botti C, et al: Predictive significance of DNA damage and repair biomarkers in triple-negative breast cancer patients treated with neoadjuvant chemotherapy: An exploratory analysis. Oncotarget 6: 42773-42780, 2015.

17. Huelsenbeck SC, Schorr A, Roos WP, Huelsenbeck J, Henninger C, Kaina B and Fritz G: Rac1 protein signaling is required for DNA damage response stimulated by topoisomerase II poisons. J Biol Chem 287: 38590-38599, 2012.

18. Li Z, Musich PR and Zou Y: Differential DNA damage responses in p53 proficient and deficient cells: Cisplatin-induced nuclear import of XPA is independent of ATR checkpoint in p53-deficient lung cancer cells. Int J Biochem Mol Biol 2: 138-145, 2011.
19. Zhong X, Luo G, Zhou X, Luo W, Wu X, Zhong R, Wang Y, Xu F and Wang J: Rad51 in regulating the radiosensitivity of non-small cell lung cancer with different epidermal growth factor receptor mutation status. Thorac Cancer 7: 50-60, 2016.

20. Tsai MS, Kuo YH, Chiu YF, Su YC and Lin YW: Down-regulation of Rad51 expression overcomes drug resistance to gemcitabine in human non-small-cell lung cancer cells. J Pharmacol Exp Ther 335: 830-840, 2010. 